\title{
BMJ Open Association of guideline and policy changes with incidence of lifestyle advice and treatment for uncomplicated mild hypertension in primary care: a longitudinal cohort study in the Clinical Practice Research Datalink
}

James P Sheppard, ${ }^{1}$ Sarah Stevens, ${ }^{1}$ Richard J Stevens, ${ }^{1}$ Jonathan Mant, ${ }^{2}$ Una Martin, ${ }^{3}$ F.D. Richard Hobbs, ${ }^{1}$ Richard J McManus ${ }^{1}$

To cite: Sheppard JP, Stevens S, Stevens RJ, et al. Association of guideline and policy changes with incidence of lifestyle advice and treatment for uncomplicated mild hypertension in primary care: a longitudinal cohort study in the Clinical Practice Research Datalink. BMJ Open 2018;8:e021827. doi:10.1136/ bmjopen-2018-021827

- Prepublication history and additional material for this paper are available online. To view these files, please visit the journal online (http://dx.doi. org/10.1136/bmjopen-2018021827).

Received 23 January 2018 Revised 2 May 2018 Accepted 25 July 2018

Check for updates

(C) Author(s) (or their employer(s)) 2018. Re-use permitted under CC BY. Published by BMJ.

${ }^{1}$ University of Oxford, 0xford, UK ${ }^{2}$ University of Cambridge, Cambridge, UK

${ }^{3}$ University of Birmingham, Birmingham, UK

Correspondence to Dr James P Sheppard; james.sheppard@phc.ox.ac.uk

\section{ABSTRACT}

Objectives Evidence to support initiation of pharmacological treatment in patients with uncomplicated (low risk) mild hypertension is inconclusive. As such, clinical guidelines are contradictory and healthcare policy has changed regularly. The aim of this study was to determine the incidence of lifestyle advice and drug therapy in this population and whether secular trends were associated with policy changes.

Design Longitudinal cohort study.

Setting Primary care practices contributing to the Clinical Practice Research Datalink in England.

Participants Data were extracted from the linked electronic health records of patients aged 18-74 years, with stage 1 hypertension (blood pressure between 140/90 and 159/99 $\mathrm{mm} \mathrm{Hg}$ ), no cardiovascular disease (CVD) risk factors and no treatment, from 1998 to 2015. Patients exited if follow-up records became unavailable, they progressed to stage 2 hypertension, developed a CVD risk factor or received lifestyle advice/treatment.

Primary outcome measures The association between policy changes and incidence of lifestyle advice or treatment, examined using an interrupted time-series analysis.

Results A total of 108843 patients were defined as having uncomplicated mild hypertension (mean age $51.9 \pm 12.9$ years, $60.0 \%$ female). Patientsspent a median 2.6 years (IQR 0.9-5.5) in the study, after which $12.2 \%$ (95\% Cl $12.0 \%$ to $12.4 \%)$ were given lifestyle advice, $29.9 \%$ (95\% Cl 29.7\% to 30.2\%) were prescribed medication and $19.4 \%$ (95\% Cl $19.2 \%$ to $19.6 \%)$ were given both. The introduction of the quality outcomes framework (QOF) and subsequent changes to QOF indicators were followed by significant increases in the incidence of lifestyle advice. Treatment prescriptions decreased slightly over time, but were not associated with policy changes.

Conclusions Despite secular trends that accord with UK guidance, many patients are still prescribed treatment for mild hypertension. Adequately powered studies are needed to determine if this is appropriate.
Strengths and limitations of this study

- This study examined electronic health records from a large, nationally representative sample of the UK population.

- Analyses were undertaken according to a prespecified protocol which was reviewed and approved by an independent scientific advisory committee.

- Mild hypertension was defined consistently according to blood pressure readings, meaning that those with a diagnostic code for hypertension but no associated blood pressure values may have been excluded.

- Low-risk patients were defined according to individual risk factors, rather than an established risk calculator due to large amounts of missing data (eg, for cholesterol, body mass index), potentially underestimating the number of truly low-risk patients available for analysis.

- The incidence of lifestyle advice was defined according to clinical codes but it was not possible to identify patients given lifestyle advice where it was either not coded or where it was only recorded in the free text consultation notes.

\section{INTRODUCTION}

High blood pressure (hypertension) is a key risk factor for the development of cardiovascular disease, ${ }^{1}$ a major cause of mortality worldwide. ${ }^{2}$ The threshold for hypertension is a sustained blood pressure $>140 / 90 \mathrm{~mm} \mathrm{Hg}$, and many guidelines recommend pharmacological treatment is initiated immediately at this level, or after a period of unsuccessful lifestyle modification, regardless of the underlying risk of cardiovascular disease. ${ }^{3-8}$ These recommendations are considered controversial, particularly with regard to treatment of uncomplicated (ie, low cardiovascular disease 
risk) people with 'mild' hypertension (ie, sustained blood pressure between 140/90 and $159 / 99 \mathrm{~mm} \mathrm{Hg}$ (stage 1)). ${ }^{9-14}$

There is little evidence to support initiation of pharmacological treatment in patients with uncomplicated mild hypertension. A Cochrane review ${ }^{15}$ of trials in this population found no reduction in mortality or cardiovascular events with treatment, but was underpowered to detect significant differences. ${ }^{10}{ }^{15}$ More recent studies ${ }^{16}{ }^{17}$ have shown benefit with treatment in patients with mild hypertension. However, these included a significant proportion of patients with diabetes or at least one other cardiovascular risk factor and therefore participants would not be considered 'uncomplicated' under current clinical guidelines. $^{18}$

Clinical guidelines often make recommendations based on 'expert opinion' where evidence is lacking or inconsistent. Such guidelines are predictably contradictory, with those in the UK promoting lifestyle modification in low-risk patients ${ }^{18}$ while guidelines from Europe ${ }^{6}$ and the $\mathrm{USA}^{478}$ encourage prescription of drug therapy. Recently, an international expert consultation recommended early initiation of blood pressure-lowering treatment with lifestyle advice reserved only for patients with grade 1 isolated hypertension. ${ }^{14}$ Against this background, a number of guideline and policy changes have occurred in the UK over the past 10 years which may have affected general practitioner's (GP's) treatment decisions. In particular, the introduction of a nationwide cardiovascular risk screening programme (the National Health Service (NHS) health check) in 2009, ${ }^{19}$ and pay-for-performance indicators (the quality and outcomes framework (QOF)), which promoted both more lifestyle advice and stricter treatment targets in this population. ${ }^{20}$

The aim of this study was to use routine patient records from the Clinical Practice Research Datalink $(\mathrm{CPRD})^{21}$ to establish the proportion of patients given lifestyle advice and/or pharmacological treatment for uncomplicated mild hypertension in primary care and the association of policy with changes to these proportions over time.

\section{METHODS \\ Design}

This was a longitudinal cohort study in conducted in Primary Care from 1998 to 2015 using data from the CPRD, a database of electronic health records from England..$^{21}$ The incidence of lifestyle advice and treatment prescriptions was estimated by month, adjusted for age and sex. The association of guideline and policy changes was examined using an interrupted time-series analysis with introduction of the NHS health check ${ }^{19}$ and changes in QOF indicators ${ }^{20}$ defined as prespecified break points. The CPRD population has been shown previously to approximate to the UK population. ${ }^{21}$

\section{Study population}

Individual patient data were extracted from the medical records of all patients registered at general practices contributing to the CPRD in England. Patients were entered into the study on the date of the third consecutive blood pressure reading between 140/90 and 159/99 mm $\mathrm{Hg}$ occurring after the study start date 1 January 1998. The start date was chosen because it represents the date from which all relevant data linkages were first available. Patients exited the study if they had three consecutive blood pressure readings $>160 / 100 \mathrm{~mm} \mathrm{Hg}$ (ie, progressed to stage 2 hypertension), developed a risk factor for cardiovascular disease, received lifestyle advice or treatment or follow-up records became unavailable (patient or practice left the CPRD) (table 1). The last day of follow-up for those remaining in the study was 30 September 2015 (last day of follow-up in linked data).

\section{Outcomes}

The primary outcome of this study was evidence that lifestyle advice and/or pharmacological treatment had been given during follow-up. Lifestyle advice was defined as Read code for any lifestyle advice relating to smoking, alcohol, diet, hypertension, physical activity, weight, oral health or lipid disorders (see online supplementary appendix etable 1). Pharmacological treatment was defined as a coded prescription of an ACE inhibitor, angiotensin II receptor blocker, calcium channel blocker, thiazide or thiazide-like diuretic, beta-blocker, alphablocker or any other antihypertensive listed in the British National Formulary (see online supplementary appendix etable 2).

\section{Covariates}

Data relating to baseline patient characteristics (age, sex, ethnicity, (practice level) deprivation), lifestyle factors (smoking status (never, current, ex-smoker), alcohol consumption (units per week), and body mass index $(\mathrm{BMI})$ ), pretreatment blood pressure readings (in the preceding 12 months), related comorbidities (rheumatoid arthritis, hypercholesterolaemia (Read code for hypercholesterolaemia or most recent total cholesterol value $>7.5 \mathrm{mmol} / \mathrm{L})$ ) and all prescribed statin/antiplatelet medications were extracted from the CPRD database and used to define the study population.

\section{Quantitative data}

Ethnicity was classified into white, black, South Asian, mixed race, other and unknown groups. The Index of Multiple Deprivation (IMD) was provided as quintiles of deprivation in England, with the highest quintile indicating the highest level of deprivation. Smoking status was defined as never, previous or current smoker. Those with unknown smoking status were assumed to have never smoked. Where there was no record of blood pressure lowering, statin or antiplatelet treatment, it was assumed that patients were not prescribed treatment. 
Table 1 Study entry and exit criteria applied to potential participant records used in the study

\section{Study entry criteria}

Date of the third consecutive blood pressure reading between $140 / 90$ and $159 / 99 \mathrm{~mm} \mathrm{Hg}$ (within 12 months of each other)

Aged between 18 and 74 years

Linked general practice, Hospital Episodes Statistics and Office for National Statistics mortality records

Registered to a CPRD practice classified as 'up-to-standard' Classified as a CPRD 'acceptable patient'

No Read code for previous cardiovascular disease (stroke, myocardial infarction, angina, coronary heart disease, peripheral vascular disease, heart failure)

No Read code for cardiovascular risk factor (left ventricular
hypertrophy, atrial fibrillation, diabetes or chronic kidney disease
or family history of premature heart disease)

No record of any blood pressure lowering medication prescription in the 12 months prior to the third consecutive blood pressure reading between 140/90 and 159/99 $\mathrm{mm} \mathrm{Hg}$

\section{Study exit (first to occur)}

Last date at which the most recent linked data are available from the CPRD (study end date, September 2015)

Date of third consecutive blood pressure reading $>160 / 100 \mathrm{~mm} \mathrm{Hg}$ (within 12 months of each other) (progression to stage 2 hypertension)

Date of first record of a cardiovascular risk factor (left ventricular hypertrophy, atrial fibrillation, diabetes or chronic kidney disease or family history of premature heart disease) or high cardiovascular risk score $(>20 \%)$

\section{Date of death}

Date of the most recent data upload from the practice to which a given patient is registered

Date at which a given patient transfers out of a registered CPRD practice

Date of first coded lifestyle advice given after study entry (lifestyle advice analysis only)

Date of first prescribed antihypertensive treatment given after study entry (treatment analysis only)

CPRD, Clinical Practice Research Datalink.

Only patients with records deemed 'acceptable for research purposes' by CPRD were included in the analysis, and hence age and sex information was complete. Where there was no record of alcohol consumption, rheumatoid arthritis or hypercholesterolaemia, patients were assumed to be non-drinkers and have no history of these conditions. Where there was no record of blood pressure lowering, statin or antiplatelet treatment, it was assumed that patients were not exposed to any treatment. A total of 91 patients $(0.001 \%)$ were missing IMD data and were excluded. To enable calculation of a cardiovascular risk score for sensitivity analyses, missing values for cholesterol and BMI were simulated once, assuming a normal distribution, and using means and SD in each age-sex strata from the 2011 Health Survey for England. ${ }^{22}$

\section{Sample size}

As a descriptive study, no formal sample size calculation was required. All patients from the CPRD fulfilling the eligibility criteria were included.

\section{Statistical analysis}

Descriptive statistics were used to describe the study population and estimate the proportion of patients being offered lifestyle advice or therapy (or both) following a diagnosis of uncomplicated mild hypertension.

The impact of the introduction of the NHS health check programme in April 2009 ${ }^{19}$ (month 111) and changes to QOF indicators (April 2009 and 2013 (month 159) $)^{20}$ (see table 2) on lifestyle advice and treatment prescription rates were examined using an interrupted time-series analysis. A 2-year run-in phase (1998-1999) was used, where patients could enter and leave the cohort, but incidence rates were not estimated. This was to ensure the cohort accumulated sufficient numbers of patients to enable accurate estimates of monthly incidence in the subsequent time period after the analysis start date (1 January 2000). The slope of the monthly rate of lifestyle advice and treatment over time was estimated (189 months in total), before policy change was introduced (months 111 and 159), along with the difference in the starting level (intercept) and slope of this rate in the period after each policy change was introduced, using ordinary least-squares regression with Newey-West corrected SEs (which account for autocorrelation). Estimates were adjusted for age and sex and all slopes are presented as incidence per year. $\mathrm{P}$ values indicate significance of the difference between the slope/intercept in the post-policy period, compared with estimates from the pre-policy period.

As a post hoc analysis, the impact of the introduction of the QOF in $2004^{23}$ (month 59) was explored as an additional breakpoint in the interrupted time-series analysis. Sensitivity analyses explored the impact of excluding those deemed to be at high risk due to (1) high cardiovascular risk score documented in the primary care physician notes and (2) high cardiovascular risk score documented in the notes or estimated using the QRISK2 algorithm ${ }^{24}$ and available risk factor data.

\section{Data access and cleaning methods}

SS had access to the entire CPRD database from which eligible patients were identified and all data extracted. 


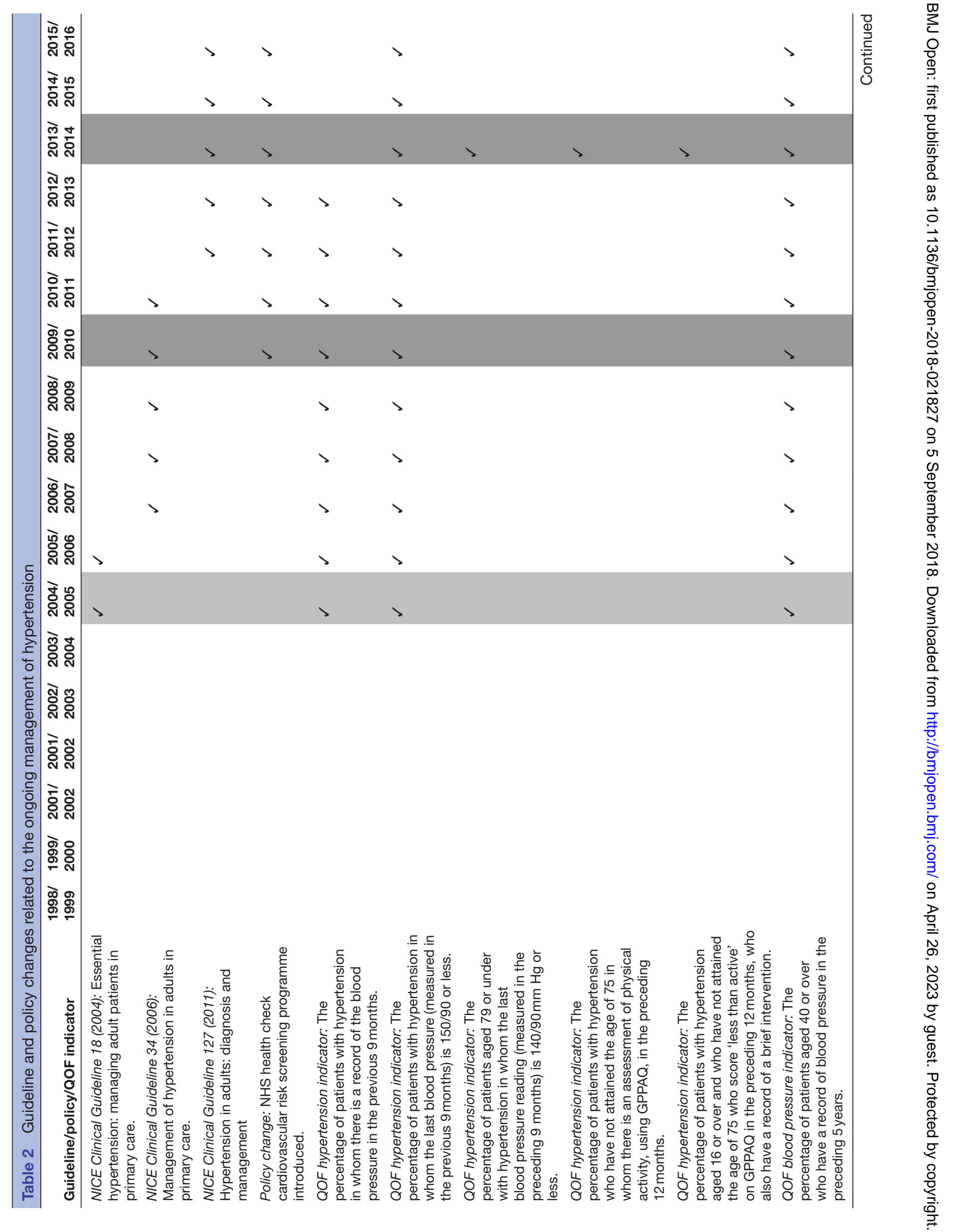




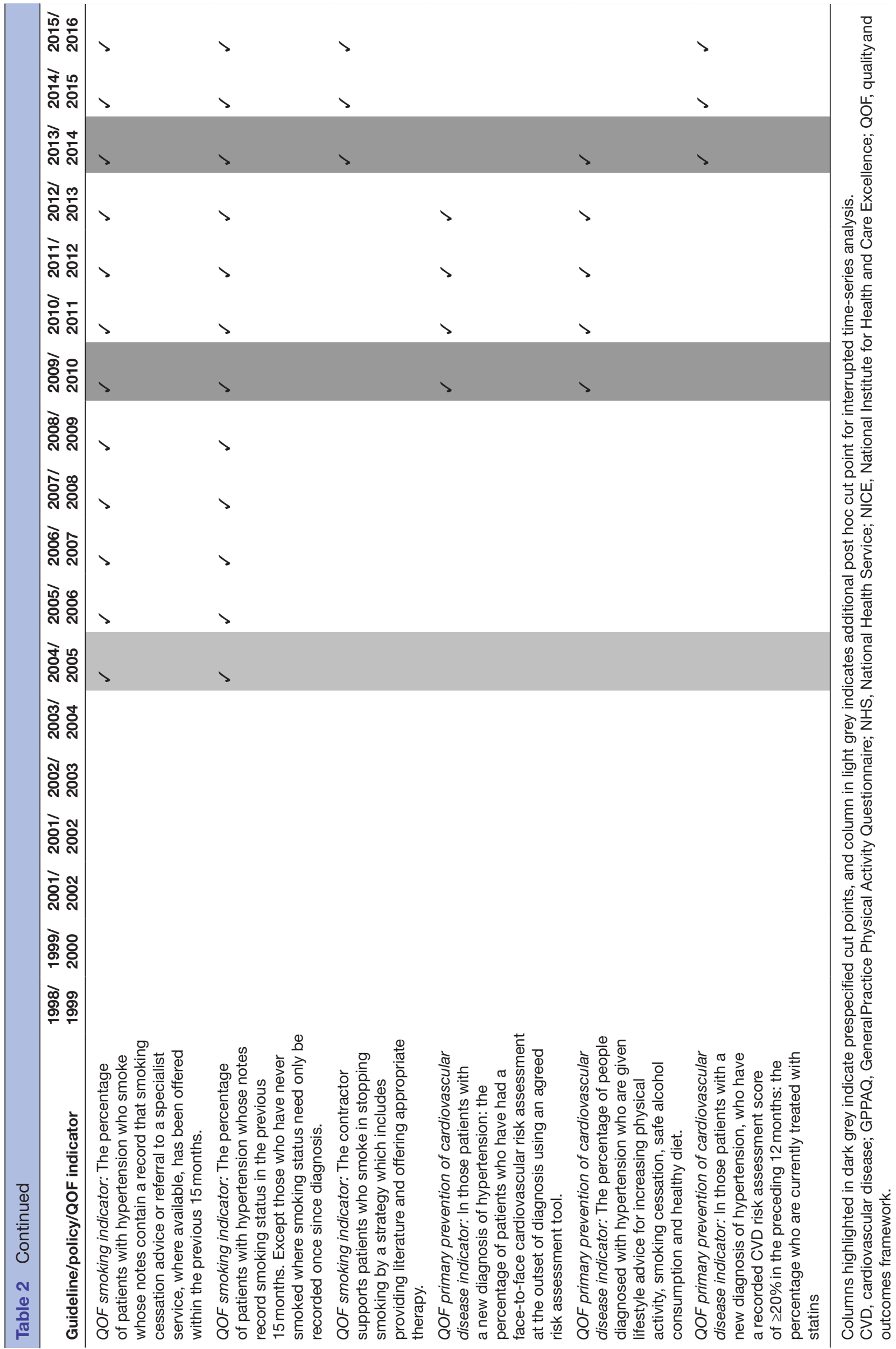


Where multiple blood pressure values appeared in the record on any oneday, the lowest value was selected. Treatments/diagnoses of conditions were determined from the presence of at least one coded prescription/ diagnostic code, respectively. Baseline data were extracted from within each patient's up to standard registration period and were drawn from the latest available record prior to study entry. Values deemed a priori to be clinically implausible were excluded (eg, weight values of $<20 \mathrm{~kg}$ and $>200 \mathrm{~kg}$ ). Where there was evidence of prior smoking but the most recent smoking status recorded indicated no smoking, this was corrected by classifying patients as former smokers.

\section{Measures taken to reduce bias}

To reduce the possibility of selection bias, all patients fulfilling the eligibility criteria for the study were included. Mild hypertension was defined according to measured blood pressure values rather than diagnostic codes, to reduce the potential for reporting bias, particularly in the pre-QOF era when coding was less standardised. For the primary analysis, breakpoints for the interrupted time-series analysis were prespecified based on policy changes thought likely to influence the giving of lifestyle advice and treatment, rather than trends observed in the data.

\section{Data linkage}

Data linkage to the Office for National Statistics (ONS) mortality register was used to define patient mortality (patient exit from the study). Linkages to Basic Inpatient Hospital Episode Statistics (HES) were used in conjunction with CPRD records to define patient eligibility for the study (eg, previous stroke), and define patient ethnicity (where unavailable in primary care records). A linkage to the IMD was required to acquire practice level indices of multiple deprivation.

\section{Patient involvement}

Patients were not involved in the development or design of this study.

\section{Approval}

The present study protocol (16_008R) was approved by this committee in March 2016, prior to obtaining the data cut (protocol given in the online supplementary appendix). A project summary was published on the CPRD website (https://www.cprd.com/isac).

All data cleaning and analyses were conducted using STATA V.13.1 and 14.2 (MP parallel edition, StataCorp). Results are presented as means, medians or proportions, with $\mathrm{SD}$, IQR or $95 \%$ CIs, unless otherwise stated.

\section{RESULTS}

\section{Prevalence of lifestyle advice and treatment}

Of the 7416968 individuals aged $18-74$ years registered at linked, up-to-standard practices within the CPRD during the study period, a total of 108843 patients $(1.46 \%, 95 \%$ CI $1.45 \%$ to $1.47 \%)$ from 694 practices $(333 \pm 192$ patients per practice) met the criteria for having uncomplicated mild hypertension during the study period (figure 1). Included patients had a mean age of $51.9 \pm 12.9$ years, $60.0 \%(95 \%$ CI $59.7 \%$ to $60.3 \%$ ) were female and mean blood pressure (across three visits) was $144.3 \pm 5.9 / 87.3 \pm 5.8 \mathrm{~mm} \mathrm{Hg}$ (table 3 ). Patients spent a median time of 2.6 years in the study (IQR 0.9-5.5) and exited the cohort due to development of stage 2 hypertension (6269 patients (5.8\%)), a cardiovascular risk factors or score $>20 \%$ (3496 patients $(5.0 \%))$, advice being given (18647 patients $(17.1 \%)$ ), treatment prescribed (35305 patients $(32.4 \%)$ ), end of study follow-up (38515 patients $(35.4 \%)$ ) or a combination of reasons (4656 patients (4.3\%)). During the study period, a total of $13269(12.2 \%, 95 \%$ CI $12.0 \%$ to $12.4 \%)$ were given lifestyle advice alone, 32578 (29.9\%, 95\% CI $29.7 \%$ to $30.2 \%$ ) were only prescribed an antihypertensive and $21128(19.4 \%, 95 \% \mathrm{CI} 19.2 \%$ to $19.6 \%)$ were given lifestyle advice and prescribed treatment (table 3). The most common type of lifestyle advice given was related to smoking (20.6\%, $95 \%$ CI $20.4 \%$ to $20.9 \%$; online supplementary appendix etable 3 ).

\section{Interrupted time-series analysis}

In the pre-NHS health check period from January 2000 to April 2009, the proportion of patients receiving lifestyle advice increased from zero by $0.04 \%$ per year $(95 \%$ CI $0.04 \%$ to $0.05 \%$ ) (figure $2 \mathrm{~A}$ ). The introduction of the NHS health check and new QOF indicators in April 2009 was associated with a significant drop in lifestyle advice being given $(p<0.001)$, but thereafter it increased by $0.19 \%$ per year ( $95 \%$ CI $0.15 \%$ to $0.23 \%$ ). Following changes to QOF indicators in April 2013, the incidence of lifestyle advice decreased by $-0.27 \%$ per year $(95 \%$ CI $-0.35 \%$ to $-0.19 \%)$.

Treatment prescriptions were given to approximately $1.29 \%$ (95\% CI $1.23 \%$ to $1.34 \%$ ) of patients with uncomplicated mild hypertension in the first month of the study, but the incidence then decreased by $-0.05 \%$ (95\% CI $-0.06 \%$ to $-0.05 \%$ ) per year in the pre-policy period until 2009 (figure 2B). Neither the introduction of the NHS health check, nor changes to QOF indicators significantly affected the incidence of treatment prescriptions which decreased by $-0.03 \%$ per year and then $-0.10 \%$ per year in the post NHS health check and QOF indicator change periods, respectively (figure 2B).

\section{Post hoc analysis}

The introduction of the QOF and clinical guidelines for the management of hypertension in 2004 was associated with a step-change increase in the incidence of lifestyle advice of $0.15 \%$ (95\% CI $0.05 \%$ to $0.26 \% ; \mathrm{p}=0.006$ ) (figure 3A). Subsequently, there was a small but significant decrease in the incidence of lifestyle advice $(-0.05 \%$ per year, $95 \%$ CI $-0.07 \%$ to $-0.04 \%)$. The NHS health check and new QOF indicators in 2009 were no longer associated with a significant drop in lifestyle advice when the introduction of the QOF (in 2004) was included as a cut-point in the post hoc analysis $(\mathrm{p}=0.07)$. No policy 


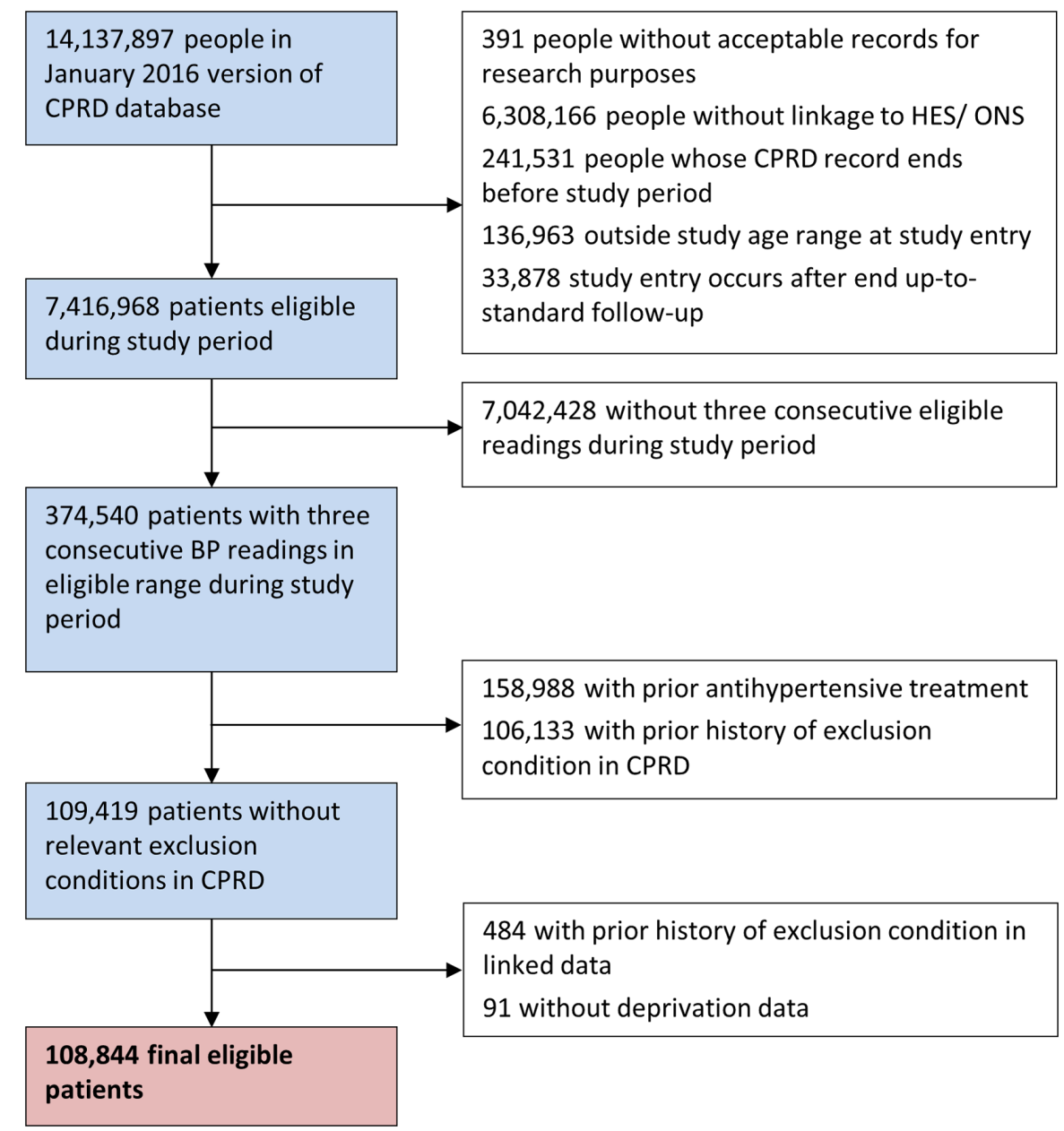

Figure 1 Flow diagram showing exclusion of patient records and definition of the final cohort. BP, blood pressure; CPRD, Clinical Practice Research Datalink; HES, Hospital Episode Statistics; ONS, Office for National Statistics.

or guideline changes were associated with significant changes in the incidence of treatment prescriptions for uncomplicated mild hypertension during the study period in the post hoc analysis (figure 3B). Exclusion of high-risk patients (using either definition) in the sensitivity analysis had no impact on the incidence of lifestyle advice or treatment prescription.

\section{DISCUSSION}

The present study examined a large database of electronic health records to establish the incidence of guideline recommended lifestyle advice and non-guideline recommended treatment for uncomplicated mild hypertension in primary care. Across the study period, approximately one-third of patients received lifestyle advice while half were prescribed antihypertensive treatment. The introduction of pay-for-performance indicators in $2004,{ }^{23}$ and more recently a cardiovascular risk screening programme, ${ }^{19}$ was associated with an increase in the proportion of patients documented as having been offered lifestyle advice, suggesting a more guideline based approach to the management of uncomplicated mild hypertension. However, there remains an appreciable proportion offered pharmacological treatment and this seems to be unaffected by changes to guidelines and policy.

\section{Strengths and limitations}

This study examined electronic health records from a large, nationally representative sample of the UK population. ${ }^{21}$ Participating practices included those which adhere to data quality standards set out by the CPRD to ensure accuracy of data studied. Analyses were undertaken according to a prespecified protocol (see online supplementary appendix) which was reviewed and approved by an independent scientific advisory committee, minimising the possibility of reporting bias and data mining.

In an attempt to define mild hypertension consistently across the population, it is possible that some patients with a diagnostic code for hypertension but no associated blood pressure values may have been excluded. The focus of this study was on 'uncomplicated' low-risk patients, but for pragmatic reasons, no attempt was made in the primary analysis to exclude those with a high risk of cardiovascular disease according to an established risk calculator ${ }^{24} 25$ due to large amounts of missing data (eg, for cholesterol, BMI). Patients did exit the cohort if 


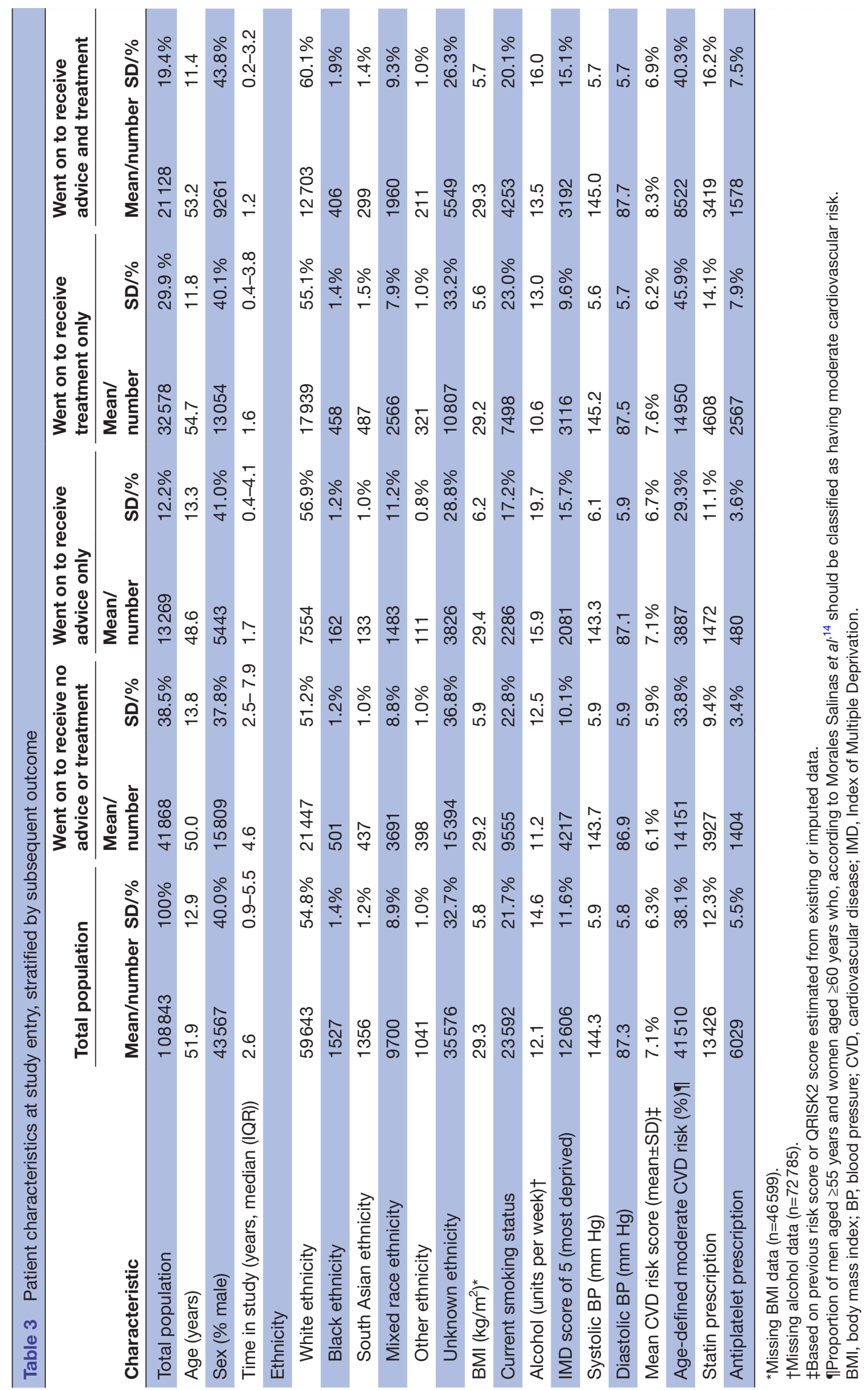




\section{A Lifestyle advice}

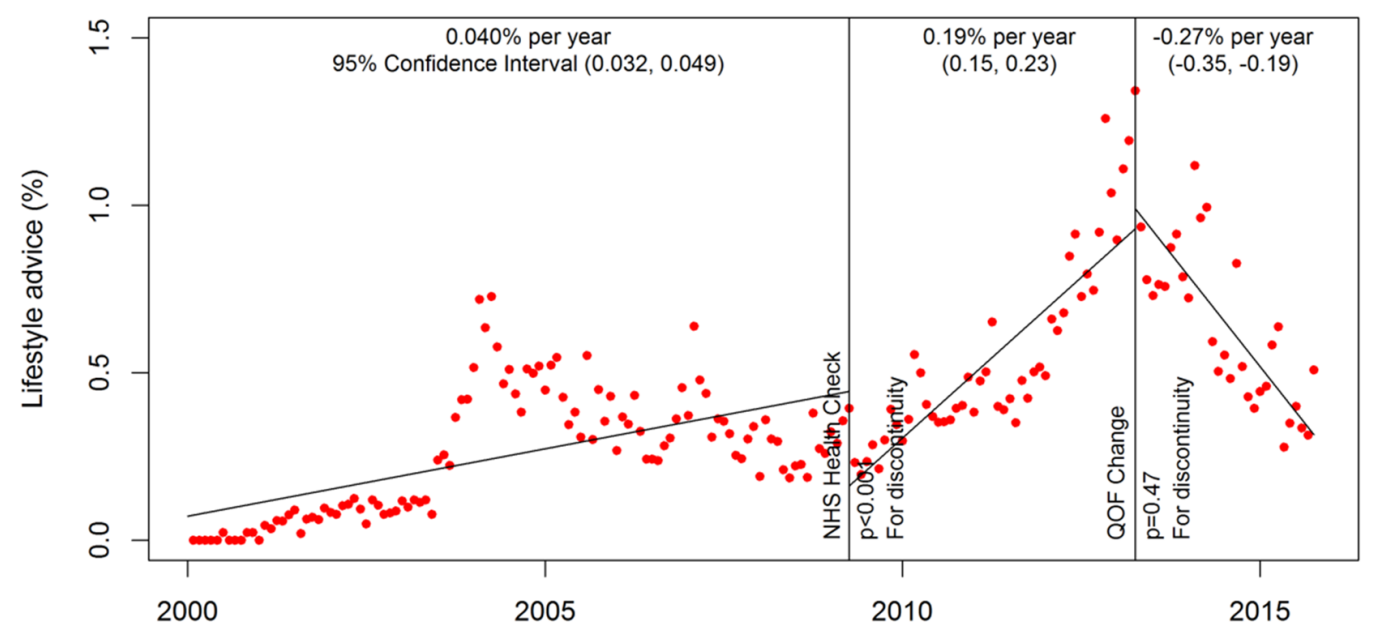

B Treatment

Month

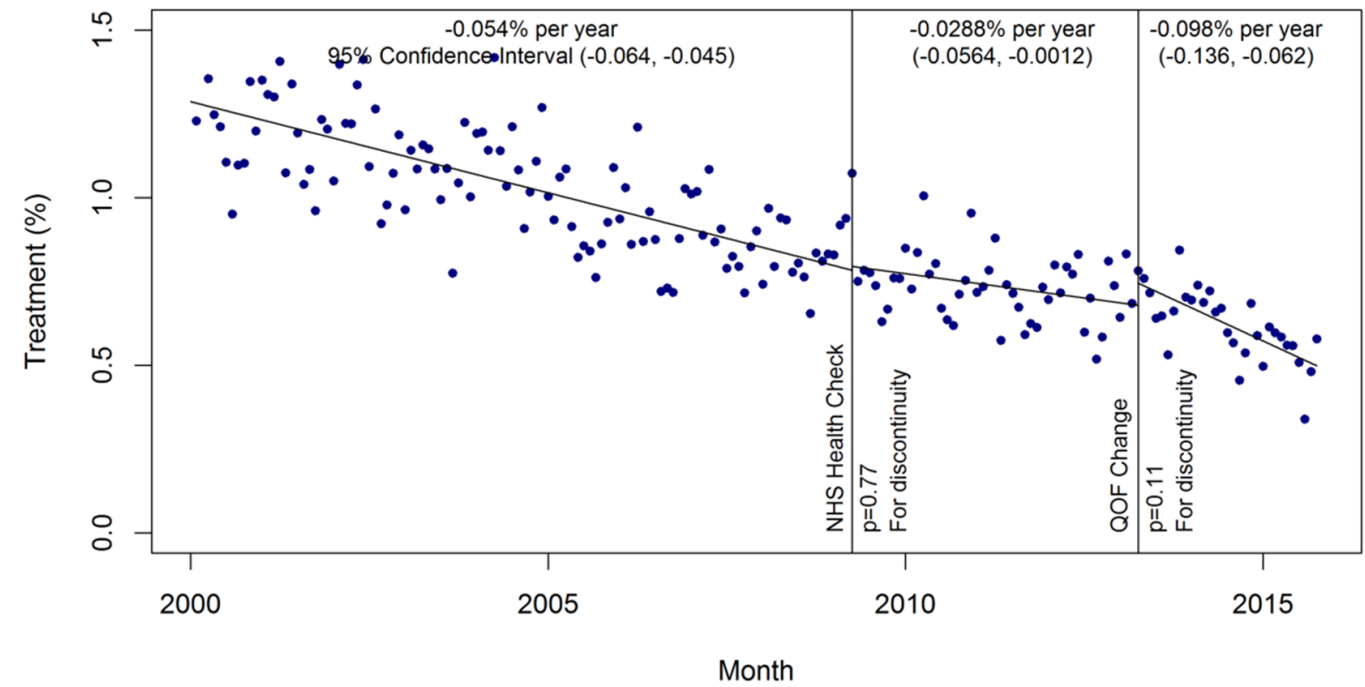

Figure 2 Primary analysis: incidence of lifestyle advice and treatment by month. (A) Lifestyle advice; (B) treatment Incidence estimates adjusted for age and sex. NHS, National Health Service; QOF, quality and outcomes framework.

they had a coded high cardiovascular disease risk score during follow-up. While it is possible that some high-risk patients may have been included in the study cohort initially, sensitivity analysis excluding patients with known high-risk scores and those with a high estimated QRISK2 score $^{24}$ did not affect the study results.

The incidence of lifestyle advice was defined according to clinical codes related to the giving of lifestyle advice which might be of relevance to the management of hypertension and cardiovascular disease risk (see online supplementary appendix etable 1). It was not possible to identify patients given lifestyle advice where it was either not coded or where it was only recorded in the free-text consultation notes. The trend of increasing lifestyle advice coinciding with the introduction of the QOF suggests that it may have been under-recorded, particularly in the early observational period.

\section{Previous literature}

To our knowledge, this is the first study to determine the incidence of lifestyle advice and treatment in patients with uncomplicated mild hypertension. Previous studies ${ }^{26-30}$ have focused on the prevalence of hypertension (defined as a blood pressure $>140 / 90 \mathrm{~mm} \mathrm{Hg}$ ) and whether or not patients are treated and controlled, without making the important distinction between hypertensive patients where treatment is appropriate and those where it is not, at least according to national guidelines. ${ }^{18}$ These surveys estimate the prevalence of hypertension across the world at between $20 \%$ and $45 \%$ with estimates of treatment and control ranging from 55\%-81\% and 30\%-59\%, respectively. ${ }^{29}$ Our data suggest that in uncomplicated mild hypertension treatment rates are slightly lower $(49 \%$ across the study period), perhaps reflecting uncertainty around its efficacy in this population. 
A Lifestyle advice

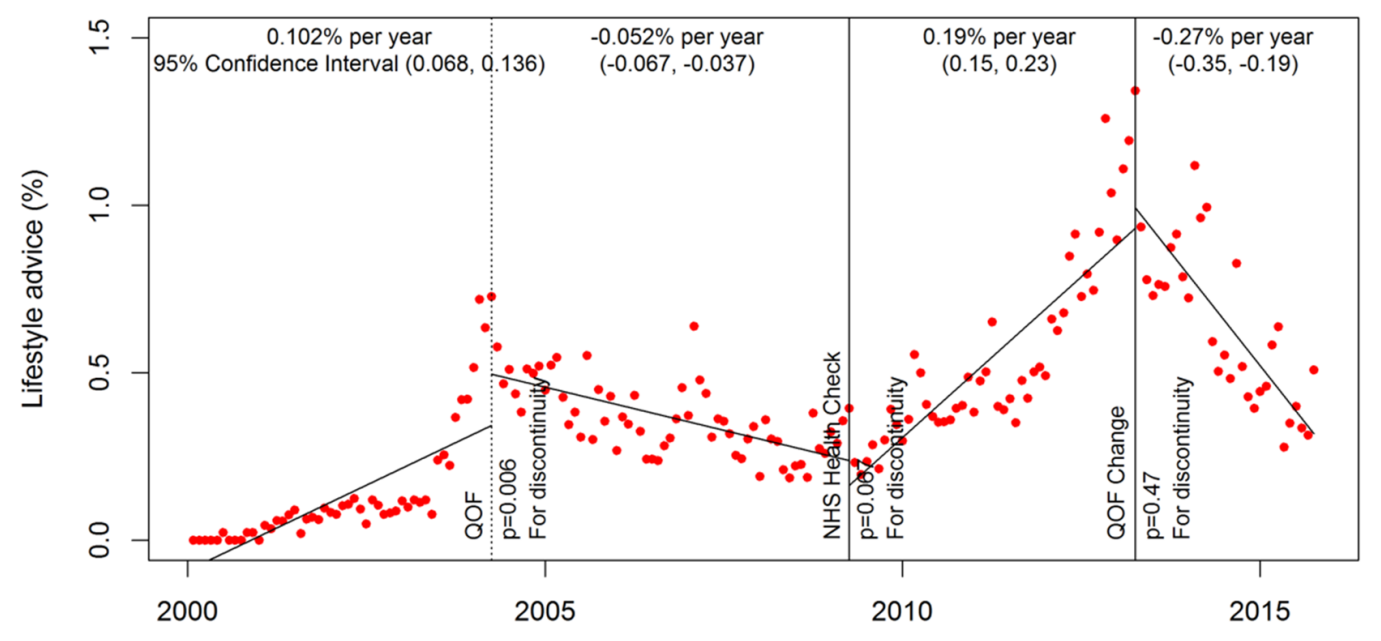

B Treatment

Month

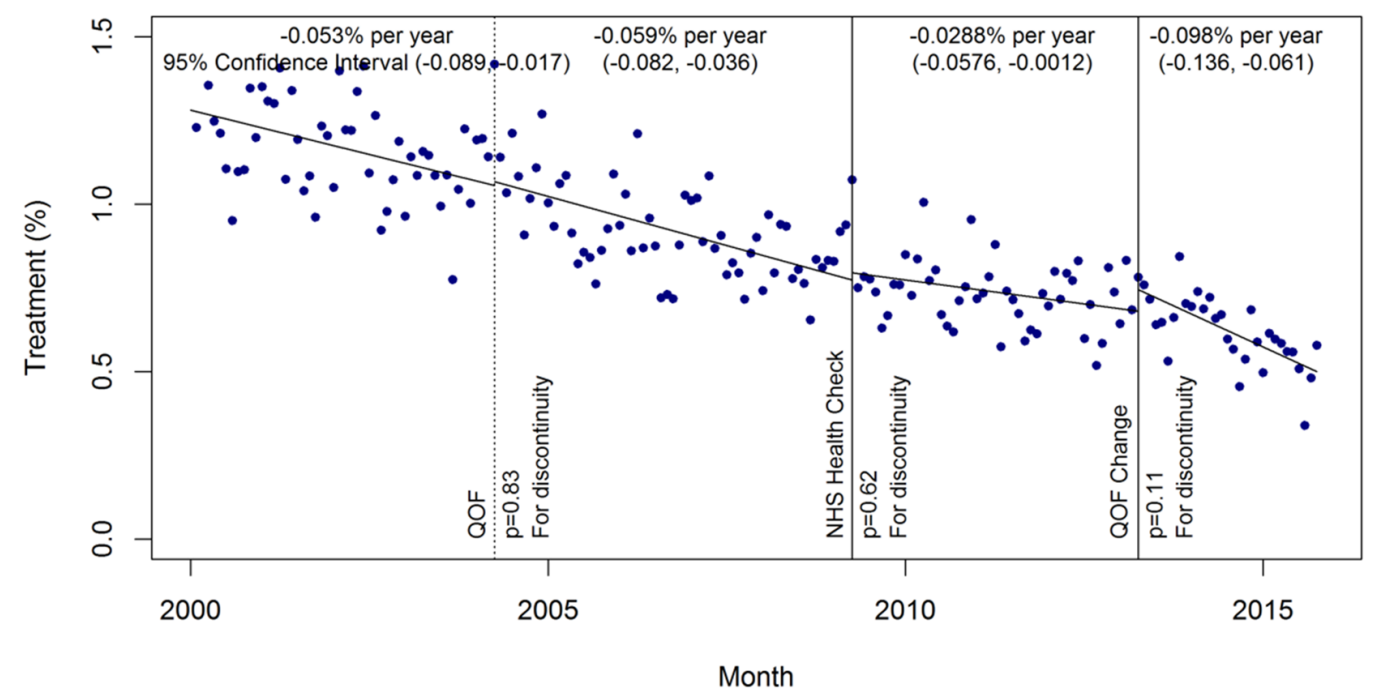

Figure 3 Post hoc analysis: incidence of lifestyle advice and treatment by month. (A) Lifestyle advice; (B) treatment Incidence estimates adjusted for age and sex. NHS, National Health Service; QOF, quality and outcomes framework.

We found a trend towards increased recording of lifestyle advice over time, particularly following the introduction of the QOF in $2004,{ }^{23}$ the NHS health check $^{19}$ and changes in QOF indicators in 2009. Previous studies examining the impact of the NHS health check have found no effect on the estimated prevalence of hypertension, ${ }^{31}$ but in those screened, referrals to specialist clinics and programmes for lifestyle advice increased sixfold. ${ }^{32}$ The apparent association between incidence of lifestyle advice and changes in QOF indicators is in contrast to previous studies which have suggested that pay-for-performance in the UK has not affected the quality of care for hypertension. ${ }^{33}$ While previous data have indicated that equivalent quality care remains, even after an indicator has been removed, ${ }^{34}$ the present data suggest the opposite, with the incidence of recording of lifestyle advice decreasing after the indicator encouraging this was removed in $2014 .^{35}$
The incidence of treatment prescriptions slightly decreased over time during the study with the largest reduction observed in the last study period (post 2013), perhaps due to more accurate blood pressure measurement and targeting of treatment brought about by new National Institute for Health and Care Excellence guidelines and the introduction of out-of-office blood pressure measurement for diagnosis in 2011. ${ }^{18}$ The overall decrease in treatment might suggest a change in GP treatment practice over the past 15 years. However, it is also possible that GP treatment practice has remained constant over time, and the number of hypertensive patients being diagnosed and perceived as low risk has increased, as evidenced by the increasing prevalence of hypertension reported annually in the QOF over the past 10 years (from $12.5 \%$ to $13.8 \%$ ). 


\section{Implications for clinical practice}

The peak in prevalence of lifestyle advice coinciding with the inclusion of QOF indicators encouraging advice for physical activity, smoking cessation and diet in $2013,{ }^{20}$ suggests that GPs were following pay-for-performance indicators closely. One explanation might be the content of QOF templates on GP computer systems, which are changed depending on the indicators included in the QOF and are likely to have encouraged better coding of lifestyle advice during a GP consultation. The choice of pay-for-performance indicators to include and exclude in the QOF should therefore be considered carefully, ensuring that such indicators reflect evidence-based practice and optimal patient care.

One could argue that the evidence for giving lifestyle advice in uncomplicated mild hypertension is as inconclusive as the evidence for pharmacological interventions, with many trials having short follow-up and exhibiting risk of bias ${ }^{18}{ }^{36}$ such as inadequate randomisation and allocation concealment, ${ }^{36}$ lack of blinding ${ }^{37}$ and incomplete outcome data. ${ }^{38}$ In addition, there are no trials of the impact of lifestyle advice on 'hard outcomes' such as cardiovascular disease and mortality ${ }^{18}$ and the quality of evidence of an association from observational studies is generally low. ${ }^{39}$ However, lifestyle advice can potentially be given at lost cost within a patient consultation, and while overall reductions in blood pressure may be modest, ${ }^{36-38}$ some patients may wish to change their lifestyle in an effort to delay or prevent embarking on a formal treatment regimen. ${ }^{36}$

\section{CONCLUSIONS}

The present study shows that the introduction of pay-for-performance indicators and a cardiovascular risk screening programme have been associated with increased guideline recommended lifestyle advice being offered to patients with uncomplicated mild hypertension, although the overall prevalence remains relatively low. However, nearly half of the patients go on to be prescribed treatment, despite a lack of evidence suggesting benefit. This may be explained by a lack of clear evidence to guide treatment decisions. Adequately powered studies, perhaps using routine clinical data at scale, are required to accurately define the relationship between treatment and cardiovascular disease and death in patients with uncomplicated mild hypertension.

Acknowledgements The authors thank Dr Blanca Gallego Luxan for reviewing and commenting on the study protocol.

Contributors JPS conceived and designed the study, secured funding, undertook the main analysis and drafted the manuscript. SS was responsible for the data management, assisted with the analysis and revised the manuscript. RJS assisted with the study design, assisted with the analysis and revised the manuscript. JM assisted with the study design and revised the manuscript. UM assisted with the study design and revised the manuscript. $\mathrm{RH}$ assisted with the study design and revised the manuscript. RJM conceived the study, assisted with the study design and revised the manuscript.

Funding This work was funded by an Medical Research Council (MRC) Strategic Skills Post-doctoral Fellowship (MR/K022032/1) held by JPS. JPS now receives funding from the National Institute for Health Research (NIHR) Collaboration for Leadership in Applied Health Research and Care Oxford at Oxford Health NHS Foundation Trust and an NIHR Professorship which is held by and also funds RJMcM (NIHR-RP- R2-12-015). FDRH acknowledges support from the NIHR as Director of the NIHR SPCR, Director of the NIHR CLARHC Oxford, Theme Leader of the NIHR Oxford BRC, NIHR Oxford DEC and also from Harris Manchester College.

Disclaimer The views and opinions expressed are those of the authors and do not necessarily reflect those of the MRC, NHS, NIHR or the Department of Health.

Competing interests RJS is a member of the CPRD's Independent Scientific Advisory Committee (ISAC), but was not involved in the approval of this study.

Patient consent Not required.

Ethics approval The present study protocol (16_008 R) was approved by this committee in March 2016, prior to obtaining the data cut (protocol given in the online supplementary appendix). A project summary was published on the CPRD website (https://www.cprd.com/isac).

Provenance and peer review Not commissioned; externally peer reviewed.

Data sharing statement Data used in this analysis was provided by the Clinical Practice Research Datalink under a Gold Licence and cannot be shared with other parties.

Open access This is an open access article distributed in accordance with the Creative Commons Attribution 4.0 Unported (CC BY 4.0) license, which permits others to copy, redistribute, remix, transform and build upon this work for any purpose, provided the original work is properly cited, a link to the licence is given, and indication of whether changes were made. See: https://creativecommons.org/ licenses/by/4.0/.

\section{REFERENCES}

1. Lewington S, Clarke R, Qizilbash N, et al. Age-specific relevance of usual blood pressure to vascular mortality: a meta-analysis of individual data for one million adults in 61 prospective studies. Lancet 2002;360:1903-13.

2. Lozano R, Naghavi M, Foreman K, et al. Global and regional mortality from 235 causes of death for 20 age groups in 1990 and 2010: a systematic analysis for the Global Burden of Disease Study 2010. Lancet 2012;380:2095-128.

3. Whitworth JA. World Health Organization, International Society of Hypertension Writing Group. 2003 World Health Organization (WHO)/International Society of Hypertension (ISH) statement on management of hypertension. J Hypertens 2003;21:1983-92.

4. Sanchez RA, Ayala M, Baglivo H, et al. Latin American guidelines on hypertension. Latin American Expert Group. J Hypertens 2009;27:905-22.

5. Ogihara T, Kikuchi K, Matsuoka H, et al. The Japanese society of hypertension guidelines for the management of hypertension (JSH 2009). Hypertens Res 2009;32:318-107.

6. Mancia G, Fagard R, Narkiewicz K, et al. 2013 ESH/ESC Guidelines for the management of arterial hypertension: the Task Force for the management of arterial hypertension of the European Society of Hypertension (ESH) and of the European Society of Cardiology (ESC). J Hypertens 2013;31:1281-357.

7. James PA, Oparil S, Carter BL, et al. 2014 evidence-based guideline for the management of high blood pressure in adults: report from the panel members appointed to the Eighth Joint National Committee (JNC 8). JAMA 2014;311:507-20.

8. Hackam DG, Quinn RR, Ravani P, et al. The 2013 Canadian Hypertension Education Program recommendations for blood pressure measurement, diagnosis, assessment of risk, prevention, and treatment of hypertension. Can J Cardiol 2013;29:528-42.

9. Toth PJ, Horwitz RI. Conflicting clinical trials and the uncertainty of treating mild hypertension. Am J Med 1983;75:482-8.

10. Tavares A. Pharmacotherapy for mild hypertension. Sao Paulo Med J 2012;130:417-8.

11. Ramsay LE, ul Haq I, Yeo WW, et al. Interpretation of prospective trials in hypertension: do treatment guidelines accurately reflect current evidence? J Hypertens Suppl 1996;14:S187-94.

12. Heath I. Waste and harm in the treatment of mild hypertension. JAMA Intern Med 2013;173:956-7.

13. Hart JT. Historical footnote on the treatment of mild hypertension BMJ 2012;345:e6297.

14. Morales Salinas A, Coca A, Olsen MH, et al. Clinical perspective on antihypertensive drug treatment in adults with 
grade 1 hypertension and low-to-moderate cardiovascular risk: an international expert consultation. Curr Probl Cardiol 2017; 42:198-225.

15. Diao D, Wright JM, Cundiff DK, et al. Pharmacotherapy for mild hypertension. Cochrane Database Syst Rev 2012;15:Cd006742.

16. Sundström J, Arima $\mathrm{H}$, Jackson R, et al. Effects of blood pressure reduction in mild hypertension: a systematic review and metaanalysis. Ann Intern Med 2015;162:184-91.

17. Wright JT, Williamson JD, Whelton PK, et al. A randomized trial of intensive versus standard blood-pressure control. N Engl J Med 2015;373:2103-16.

18. National Clinical Guideline Centre. Hypertension: clinical management of primary hypertension in adults; Clinical guideline 127. London: Royal College of Physicians (UK), 2011.

19. Public Health England. NHS health check: our approach to the evidence. 2013 https://www.gov.uk/government/uploads/system/ uploads/attachment_data/file/224537/NHS_Health_Check_our_ approach_to_the_evidence_v2.pdf

20. NHS Employers GPC, NHS England. General Medical Services (GMS) contract Quality and Outcomes Framework (QOF): guidence for GMS contract 2013/14. NHS Employers: Leeds, 2013.

21. Herrett E, Gallagher AM, Bhaskaran K, et al. Data resource profile: clinical practice research datalink (CPRD). Int J Epidemiol 2015;44:827-36.

22. Joint Health Surveys Unit. Health survey for England 2011, health and social care information centre. 2012 http://digital.nhs.uk/ catalogue/PUB09300

23. NHS Employers GPC, NHS England. General Medical Services (GMS) contract Quality and Outcomes Framework (QOF): guidence for GMS contract 2004/05. NHS Employers: Leeds, 2004.

24. Hippisley-Cox J, Coupland C, Vinogradova Y, et al. Predicting cardiovascular risk in England and Wales: prospective derivation and validation of QRISK2. BMJ 2008;336:1475-82.

25. D'Agostino RB, Vasan RS, Pencina MJ, et al. General cardiovascular risk profile for use in primary care: the Framingham Heart Study. Circulation 2008;117:743-53.

26. Joffres M, Falaschetti E, Gillespie C, et al. Hypertension prevalence, awareness, treatment and control in national surveys from England, the USA and Canada, and correlation with stroke and ischaemic heart disease mortality: a cross-sectional study. BMJ Open 2013;3:e003423.
27. Neuhauser HK, Adler C, Rosario AS, et al. Hypertension prevalence, awareness, treatment and control in Germany 1998 and 2008-11. J Hum Hypertens 2015;29:247-53.

28. Labeit AM, Klotsche J, Pieper L, et al. Changes in the prevalence, treatment and control of hypertension in Germany? A clinicalepidemiological study of 50.000 primary care patients. PLOS One 2012;7:e52229.

29. Ikeda N, Sapienza D, Guerrero R, et al. Control of hypertension with medication: a comparative analysis of national surveys in 20 countries. Bull World Health Organ 2014;92:10-19.

30. Wolf-Maier K, Cooper RS, Kramer H, et al. Hypertension treatment and control in five European countries, Canada, and the United States. Hypertension 2004;43:10-17.

31. Caley M, Chohan P, Hooper J, et al. The impact of NHS Health Checks on the prevalence of disease in general practices: a controlled study. Br J Gen Pract 2014;64:e516-21.

32. Robson J, Dostal I, Sheikh A, et al. The NHS Health Check in England: an evaluation of the first 4 years. BMJ Open 2016;6:e008840.

33. Serumaga B, Ross-Degnan D, Avery AJ, et al. Effect of pay for performance on the management and outcomes of hypertension in the United Kingdom: interrupted time series study. BMJ 2011;342:d108.

34. Kontopantelis E, Springate D, Reeves D, et al. Withdrawing performance indicators: retrospective analysis of general practice performance under UK Quality and Outcomes Framework. BMJ 2014;348:g330

35. NHS Employers GPC, NHS England. General Medical Services (GMS) contract Quality and Outcomes Framework (QOF): guidence for GMS contract 2014/15. NHS Employers: Leeds, 2014.

36. Dickinson HO, Mason JM, Nicolson DJ, et al. Lifestyle interventions to reduce raised blood pressure: a systematic review of randomized controlled trials. J Hypertens 2006;24:215-33.

37. Cornelissen VA, Smart NA. Exercise training for blood pressure: a systematic review and meta-analysis. J Am Heart Assoc 2013;2:e004473.

38. Ndanuko RN, Tapsell LC, Charlton KE, et al. Dietary patterns and blood pressure in adults: a systematic review and meta-analysis of randomized controlled trials. Adv Nutr 2016;7:76-89.

39. Aburto NJ, Ziolkovska A, Hooper L, et al. Effect of lower sodium intake on health: systematic review and meta-analyses. BMJ 2013;346:f1326. 